Courrier du Centre International Blaise-

Pascal

38-39 | 2017-2018

Varia

\title{
Cet obscur « objet de la poésie »
}

\section{Tony Gheeraert}

\section{CpenEdition}

Journals

Édition électronique

URL : https://journals.openedition.org/ccibp/1363

DOI : $10.4000 /$ ccibp. 1363

ISSN : 2493-7460

\section{Éditeur}

Centre international Blaise Pascal

\section{Édition imprimée}

Date de publication : 1 janvier 2019

Pagination : 87-105

ISBN : 978-2-84516-897-8

ISSN : 0249-6674

\section{Référence électronique}

Tony Gheeraert, «Cet obscur « objet de la poésie » », Courrier du Centre International Blaise-Pascal [En ligne], 38-39 | 2017-2018, mis en ligne le 18 juin 2021, consulté le 04 mai 2022. URL : http:// journals.openedition.org/ccibp/1363; DOI : https://doi.org/10.4000/ccibp.1363

Ce document a été généré automatiquement le 4 mai 2022.

Centre international Blaise Pascal 


\title{
Cet obscur « objet de la poésie »
}

\author{
Tony Gheeraert
}

1 Par un étrange caprice de l'histoire des textes - mais est-ce vraiment un hasard? -, c'est sur la poésie que s'ouvrent les éditions récentes des Pensées : « les psaumes chantés par toute la terre " $\left(\mathrm{S} 1^{1}\right)$. Pascal poète : le fragment 1 nous suggère une piste qui n'a encore été que peu frayée, si l'on excepte le travail fondateur d'olivier Jouslin ${ }^{2}$. Le rôle joué par la rhétorique biblique sur l'œuvre de Pascal a été bien étudié, grâce entre autres aux travaux de Philippe Sellier et de Laurent Susini : si l'apologiste ne goûte guère les fastes de l'art oratoire classique, il emprunte aux livres prophétiques de la Bible ses genres, ses formes, et ses tropes. «Dieu parle bien de Dieu », et c'est auprès du véritable Auteur du Texte sacré qu'il faut apprendre l'art d'une écriture efficace susceptible de toucher le cœur ${ }^{3}$. Mais l'imitateur attentif des prophètes a-t-il de même cherché à retrouver le secret de la poésie biblique, en tant qu'elle ne se laisse pas réduire à des figures rhétoriques mais obéit à des procédures et à une forme qui lui seraient propres? Pour répondre à cette question, nous reconstituerons la façon dont PortRoyal se représentait à la fois les enjeux et la forme de la poésie biblique, avant de montrer que cette conception port-royaliste de la poésie fournit à Pascal à la fois un paradigme gnoséologique et un art d'agréer.

2 L'existence même d'une poésie au sein des Écritures ne fut jamais une évidence qui se serait imposée d'elle-même. Les humanistes cherchèrent en vain à retrouver la prosodie ou la métrique à l'œuvre dans les livres de la Bible considérés comme "poétiques ». À la fin du XVII siècle, l'oratorien Bernard Lamy avouait encore qu'il échouait à y reconnaître les principes qui valent pour la plupart des langues anciennes ou modernes :

Pour les Hébreux, on ne sait pas très bien en quoi consistait leur poésie, quelles en étaient leurs règles. Il y en a qui pensent que leurs cantiques ne consistaient que dans des expressions nobles, et extraordinaires; néanmoins ce style des cantiques si différents de la prose, ces manières contraintes et obscures furent assujettis à garder des mesures, que nous ne pouvons pas distinguer. On y trouve bien des spondées, des ïambes, des dactyles; mais ce n'est pas assez, il faudrait que ces pieds fussent liés ensemble comme dans la poésie latine, ce qu'on n'aperçoit pas. Les Hébreux ne coupaient pas non plus leurs discours en certaines parties qui eussent 
un certain nombre de voyelles et qui finissent par une même rime, ce qui fait aujourd'hui la poésie de tous les Orientaux et de tous les Européens ${ }^{4}$.

Ce n'est qu'au milieu XvIII ${ }^{\mathrm{e}}$ siècle, en 1753 , que l'évêque Lowth proposa de résoudre cette énigme en abordant le problème sous un angle radicalement différent, celui de la syntaxe. Selon l'auteur des Leçons sur la poésie sacrée des Hébreux, en effet, le système poétique hébraïque reposait non sur un formalisme de langue (métrique ou prosodie) mais sur des phénomènes de parallélismes. L'hypothèse de Lowth est aujourd'hui largement admise, et la plupart des spécialistes se sont rangés à son point de vue : le parallélisme est le «trait déterminant du discours poétique de l'hébreu ancien », écrit ainsi Robert Alter, auteur de l'Art de la poésie biblique ${ }^{5}$. Certes, les opinions émises par les hébraïsants d'aujourd'hui nous importent sans doute moins, pour comprendre Pascal, que celles de ses contemporains et particulièrement celles qui émanaient de Port-Royal. Or, en pratique, il ne semble pas que les poètes proches du monastère aient intuitivement perçu les mécaniques propres à la poésie hébraïque, non plus que les grammairiens : il n'en est fait mention ni dans le traité de versification de Lancelot, ni dans les textes de Pierre Nicole sur la poésie ${ }^{6}$. Les préfaces de la Bible seraient-elles susceptibles de nous offrir quelques échappées sur cette question? Elles sont, certes, quelque peu postérieures à l'œuvre de Pascal, mais ce dernier a participé aux premières réunions du comité éditorial, et le paratexte qui accompagne ce monument représente davantage une vulgate couramment admise plutôt que l'opinion propre de Sacy et de ses collaborateurs.

On constate tout d'abord que les éditeurs de la Bible ne reconnaissaient pas en tant que telle l'existence d'un ensemble cohérent de «livres poétiques» aux côtés des "prophétiques » et des «historiques». La distinction, qui remonte à saint Jean Chrysostome, ne leur est pas étrangère, mais le massif qui nous apparaît aujourd'hui comme poétique leur paraissait surtout relever de la littérature morale et didactique : l'Ecclésiastique est ainsi avant tout un « livre qui prêche ", estime Sacy; ce mot, qui peut être rapproché du terme «symbouleutikon» («qui conseille») employé par le pseudoChrysostome dans son Synopsis Scripturae Sacrae, renvoie plutôt à la rhétorique délibérative qu'à la poésie. Pour ce qui est de la forme, les préfaciers considèrent comme Bernard Lamy que le secret de la versification hébraïque ancienne est perdu, et que les tentatives pour y retrouver les schémas habituels de la poésie antique ont échoué : «Joseph dit [à propos du Chant de l'Exilé] que ce sont des vers hexamètres. Mais les savants demeurent d'accord, qu'il est bien difficile de discerner la mesure des vers hébreux ", précise ainsi l'introduction de l'Exode ${ }^{7}$.

Est-ce à dire que les Messieurs ont renoncé à s'interroger sur la nature et la fonction des livres poétiques de la Bible? Non certes. Plusieurs préfaces en particulier sont l'occasion pour eux d'exposer leur conception de la poésie biblique, de sa forme, mais aussi et surtout de ses enjeux. Ils perçoivent ainsi comme poétique le "Chant de l'Exilé ", poème d'actions de grâce entonné par « Moïse et les enfants d'Israël » au sortir d'Égypte: «Ce Cantique est en vers. Et ce sont les vers les plus anciens que l'on sache avoir jamais été faits dans le monde ${ }^{8} »$. Cette dimension poétique leur apparaît également essentielle à la compréhension du Cantique des Cantiques, dont on sait qu'il embarrassait les éditeurs et qu'ils en ont reporté le plus possible la publication. Certes, ils échouaient à trouver des schémas métriques dans cette œuvre, mais ils en reconnaissaient indiscutablement la forme : "Ce Cantique est proprement un poème, mais dont les vers, pour ce qui regarde la mesure, nous sont inconnus ». La poésie de ce texte réside d'abord selon eux dans le recours aux images, dont ils attribuaient 
l'abondance à un goût oriental. La langue de ces contrées, expliquent-ils, est «si remplie de figures et de paraboles", que les discours des habitants "n'ont presque jamais rien de simple et d'un langage commun, mais sont tout entiers mêlés d'énigmes et de métaphores». Aussi, dans le Cantique, "les expressions qui paraissent hyperboliques [sont] regardées comme des manières de parler propres au pays ${ }^{9} »$. Les éditeurs justifiaient également la sensualité des images par leur outrance même: personne ne pouvait les prendre à la lettre; aussi chaque lecteur se trouvait-il invité par là à chercher, sous l'écorce du texte, sa signification spirituelle :

Beaucoup d'expressions métaphoriques, comme sont celles de parfums, baisers, cheveux, dents, cou [sont des] nuages qui couvrent [la vérité], des allégories. [...] Ce grand nombre d'expressions figurées [...] sont répandues partout dans les anciens livres de l'Écriture, et [...] étant des noms propres de certaines choses, en signifient néanmoins visiblement d'autres ${ }^{10}$.

6 L'originalité du Cantique consiste dans son absence de sens littéral : "le sens propre » de « tout le cantique » est " spirituel et divin ». Ou pour mieux dire « le vrai sens littéral regarde le mystère de l'Incarnation ${ }^{11} "$, c'est-à-dire qu'il se trouve absorbé et comme annulé dans son sens spirituel, au nom du fonctionnement même de la poésie hébraïque tel que l'entendent les Solitaires ; la dimension poétique du livre leur permet ainsi, non sans paradoxe, d'évacuer ce qu'il peut y avoir d'inconfortable pour eux dans le sens propre de ces images. Les Messieurs, dont on connaît pourtant la méfiance habituelle envers les figures de transfert, revendiquent hautement ici pour le Cantique un caractère métaphorique et " parabolique » qu'ils refusent généralement aux autres livres bibliques ${ }^{12}$. Le Cantique est allégorique, certes, mais ses figures désignent la vérité, loin de la déguiser et de la masquer. À l'inutile ornatus païen s'opposent des images dont la fonction est au contraire de révéler le message divin. Ces figures relèvent de la condescendance divine (sugkatabasis), c'est-à-dire des procédés dont Dieu use pour faire connaître ses plus profonds mystères aux hommes, dont les sens et l'intelligence sont limités.

Mais disons de plus, que le Saint-Esprit s'abaissant en quelque façon à la portée de l'intelligence des hommes, leur parle dans ce cantique d'une manière humaine, pour se faire mieux entendre à eux, comme saint Paul inspiré de lui le disait sur un semblable sujet à quelques fidèles : humanum dico, propter infirmitatem carnis vestrae: je vous parle humainement à cause de la faiblesse de votre chair. Car l'homme étant devenu par le péché animal et charnel, comme dit le même apôtre, il n'est plus capable par lui-même des choses qu'enseigne l'Esprit de Dieu. Ainsi il est nécessaire en quelque sorte, que Dieu s'abaisse jusqu'à ce qui tombe sous ses sens, pour se faire entendre à lui, et pour l'élever ensuite jusqu'aux choses de l'esprit ${ }^{13}$.

On pourrait penser que Dieu renonce à sa dignité et à sa majesté en recourant à des artifices poétiques aussi hardis. En réalité, l'évidente inappropriation de la figure à la vérité divine est une incitation pour le lecteur à prendre la mesure de sa propre indignité, qui a conduit Dieu à choisir un tel langage, si visiblement inadapté à son objet. Ce mode spécifique de communication du divin, propre aux livres poétiques, convient bien à notre état pécheur, car notre raison est impuissante à s'élever jusqu'au Ciel. Dieu, que nous ne pouvons contempler dans son essence, accepte toutefois de se laisser entrevoir à travers le voile translucide des figures, en ce monde où nous ne le découvrons que comme dans un "miroir», et à travers les "énigmes" consubstantielles de l'écriture poétique ${ }^{14}$ :

toutes ces métaphores n'étaient employées que pour faire entendre à des hommes aussi grossiers qu'étaient les Juifs, d'une manière plus sensible et plus 
proportionnée à leur esprit tout charnel, des mystères infiniment élevés au-dessus des sens ${ }^{15}$.

8 Si les images poétiques relèvent de la pédagogie divine, elles ont aussi pour tâche de celer et de sceller une sagesse qui doit rester inaccessible aux orgueilleux et aux philosophes : elle n'est réservée qu'aux humbles lecteurs et aux déchiffreurs patients. Dieu recourt aux tropes surprenants et aux obscurités plutôt qu'à des concepts en apparence clairs et distincts, afin de se conformer non à l'intelligence, mais au cœur : la "condescendance " n'est pas à entendre ici seulement au sens rhétorique d'une adaptation d'ordre psychologique ou social aux auditeurs, comme le serait "l'accommodation» de Quintilien ${ }^{16}$, mais dans une perspective épistémologique. La poésie, en voilant et dévoilant simultanément l'Être divin, permet de le connaître, autant du moins qu'il est possible ici-bas.

Les métaphores du Cantique, dans lesquels les éditeurs voient une marque du style oriental des Écritures, sont aussi selon les Messieurs l'âme de la poésie des Psaumes:

Ce qui relève encore [...] le livre des psaumes, c'est la poésie toute sainte, jointe à une multitude d'excellentes métaphores, et à un genre nouveau d'écrire, qui excite si vivement à l'amour et à la louange du Seigneur, qu'on ne peut ni rien chanter ni rien entendre de plus agréable et de plus utile ${ }^{17}$.

10 Le Psautier est le livre de la Bible où la dimension poétique apparaît le plus clairement aux Solitaires. Il ne fait pas de doute pour les exégètes que les psaumes ressortissent au lyrisme. David est à leurs yeux un « luth animé », auteur de « chants » ou de " cantiques » dont l' «harmonie toute céleste », remarquable par sa "douceur ", ne séduit pas seulement les oreilles, mais touche aussi l'esprit: David «inspire à l'âme [...] une douceur ${ }^{18} »$. Cette suavitas relève certes d'un delectare issu de la tradition d'Augustin, mais aussi et surtout du movere; elle est puissante sur les cœurs, et opère comme la grâce, agissant « par une certaine douceur [...] qui porte à la vertu ${ }^{19}$ ". Cette grâce est médicinale et thérapeutique, pour ainsi dire "cathartique » : il est en effet au pouvoir de cette poésie de guérir les âmes pécheresses et malades, ou d'apaiser leurs tourments en chassant les démons qui les assaillent, comme en témoigne la puissance orphique de la lyre de David, seule à même de calmer l'âme troublée de Saül. La poésie apparaît comme l'instrument favori de Dieu lorsqu'il entreprend de convertir les cœurs :

La psalmodie produit le plus grand de tous les biens qui est la charité [...]. Le psaume chasse les démons, nous attire le secours des anges [...]. Il produit également la joie du Saint-Esprit, et la tristesse qui est selon Dieu, puisqu'il a la force de tirer des larmes d'un cœur de pierre.

11 Les psaumes détournent même la colère divine, " apais[ant] la juste colère de Dieu dans les grandes afflictions ». Les effets de ces cantiques sont les mêmes que ceux prêtés par Port-Royal à la Charité ; on chante en effet le psautier, explique Sacy,

soit pour attirer ses grâces sur les fidèles; soit pour repousser les tentations de l'ennemi ; soit pour réveiller la foi assoupie des Chrétiens ; soit pour exciter en eux des mouvements d'une véritable pénitence; ou pour allumer au fond de leur cœur le feu de la charité. ${ }^{20}$

12 Les psaumes ne se contentent pas d'énoncer ou pour ainsi dire de prêcher les vérités de la foi : ils confèrent la force de l'accomplir, ils « inspirent un désir ardent » de respecter les commandements ${ }^{21}$, comme opère aussi la grâce du Christ. Les psaumes nous entraînent quasi invinciblement, ils nous charment parce que, comme carmina, ils sont des incantations capables de venir à bout des résistances de notre âme, et de nous mettre en état de goûter "la beauté si charmante de la loi de Dieu». Ce "plaisir 
extraordinaire ${ }^{22}$ ", que nous font sentir les psaumes, c'est la puissance efficace de l'Esprit; comme celui-ci est notre Paraclet, ceux-là sont nos « consolateurs » : ainsi que le disait Chrysostome, du "chant des psaumes", "vous en tirerez une grande consolation ", rappelle le préfacier ${ }^{23}$. La joie qu'ils procurent n'a rien à voir avec celle que donnent les chansons impudiques de la poésie profane : le «plaisir très chaste » des psaumes participe au contraire de la delectatio victrix qui incline au bien et à l'amour de la justice. On conçoit l'attention toute particulière que les Messieurs ont accordée à ce livre $^{24}$ : la poésie biblique des psaumes, qui émane du Saint-Esprit, lui emprunte sa puissance et son efficace : « le Saint-Esprit descend [dans l'âme] dans le moment qu'elle chante ses chants sacrés ${ }^{25}$."

Pour les Solitaires, les psaumes se définissent d'abord par leur nature figurative, bâtie sur des «métaphores ", des " énigmes » et des «allégories » : leur poésie est «toute sainte, jointe à une multitude d'excellentes métaphores ${ }^{26} »$. Dissimulée sous le voile des obscurités, c'est toute une «théologie parfaite ${ }^{27}$ » qui s'y laisse percevoir au prix d'un patient déchiffrage, car les psaumes contiennent, comme un cœur celé sous l'écorce de la lettre, tous les mystères de la religion:

On y trouve les prophéties touchant l'Incarnation du Fils de Dieu; les menaces du jugement ; l'espérance de la Résurrection ; la crainte des châtiments ; les promesses de la gloire; la révélation de tous les mystères; comme en un trésor qui renferme tous les véritables biens ${ }^{28}$.

Les figures ont pour tâche de dissimuler les secrets divins, afin que ceux-ci restent imperceptibles aux yeux des superbes et des mondains; ces versets demeurent

comme scellés à l'égard des hommes remplis de la prudence du siècle, pour ne les pas exposer à la vue de ceux que leur orgueil ou leur incrédulité rendrait indignes de les connaître ${ }^{29}$.

Mais cette offuscation de la lumière divine procède aussi, ici encore, de cette condescendance épistémologique et pédagogique de la majesté divine qui constitue la principale justification des obscurités: les psaumes constituent en effet «un assemblage des maximes les plus pures et les plus proportionnées à nos besoins $\mathrm{s}^{30}$ \%

Il existe une exception à cette règle générale de la lecture figurative: celle du psaume 118, cher entre tous à Pascal, et dont la particularité consiste précisément d'être un "psaume sans figures ${ }^{31}$ ». Le Saint-Esprit qui inspire ce poème opte ici pour une voie sans détour : il « ne couvrait d'aucunes énigmes ni d'aucuns voiles les vérités qu'il annonçait». L'analyse formelle dont fait l'objet ce texte est d'autant plus précieuse pour nous qu'aux yeux des Messieurs, sa poésie ne procède pas d'une rhétorique typologique. Ce n'en est pas moins un poème, insistent les éditeurs, qui s'attardent exceptionnellement à considérer le fonctionnement de ces "versets ». Ceux-ci sont définis comme «des sentences courtes qui renferment les plus grandes vérités de la morale », ou comme l'expression de "mouvements très-vifs d'une charité ardente "; ou encore comme des "prières d'un cœur pénétré de sa faiblesse ou de sa misère »; le psaume se caractérise aussi par sa tonalité, élégiaque (il peut exprimer les "regrets d'une âme percée de douleur par le souvenir de ses péchés») ou encomiastique ( des éloges et des admirations perpétuelles»). Enfin, les préfaciers notent que la forme des psaumes de David en facilite la mémorisation:

Il arrive rarement que quelqu'un du peuple et de ceux qui sont négligents pour toutes les choses divines, retienne en sortant de l'Église quelque parole des épîtres des Apôtres ou des prophètes. Au lieu qu'ils chantent souvent dans leurs maisons des versets des psaumes ${ }^{32}$. 
17 Le commentateur ne précise pas ici si cette facilité des cantiques à s'imprimer dans la mémoire procède des phénomènes de répétition qui structurent le psautier ; mais on a vu que le procédé de reprise avait été formellement identifié à propos du chant de l'Exilé.

18 Les préfaciers concluent avec saint Ambroise sur la valeur sacrée entre tous du livre des psaumes, et sur le caractère exceptionnel de son écriture; si les livres historiques sont semés de quelques cantiques qui en sont comme les "ornements ", la poésie est consubstantielle au psautier, et lui confère une élévation qui confine au sublime : il est «tout composé de Cantiques sacrés qui ont quelque chose de plus élevé que le reste de l'Écriture ». La brièveté et la densité distinguent en effet les psaumes des livres « historiques » ou « prophétiques », ou encore d'autres livres comme celui de Job :

Tout ce que Moïse a rapporté dans son Histoire; ou établi par la loi ; et tout ce que les autres prophètes ont écrit, ou pour exhorter à la vertu, ou pour prédire les choses futures, le saint roi David l'a renfermé dans ses psaumes d'une manière courte et très élevée ${ }^{33}$.

19 Les éditeurs sont bien plus discrets sur les autres livres que nous classerions volontiers comme poétiques. Mais même si les termes de poésie et de vers ne sont pas mentionnés dans la préface du Livre des Proverbes, les difficultés du texte amènent Sacy à mettre en place un type de lecture et d'interprétation qui correspond à un fonctionnement du texte qu'on peut définir comme poétique. Les Proverbes sont ainsi d'abord conçus comme des allégories, des "paraboles »- le mot était parfois employé comme titre de cet ouvrage. Ces allégories se définissent par leur style sentencieux et le recours à une forme de théologie naturelle. Ils forment un

livre des sentences graves et divines, qui sont souvent mêlées de quelques obscurités, et de comparaisons prises de ce qui se passe dans la nature, où Dieu rend comme sensibles les choses les plus spirituelles et cachées ${ }^{34}$.

Préfaciers et approbateurs soulignent l'opacité rebutante du texte. L'évêque d'Alet Nicolas Pavillon écrit ainsi au moment d'accorder son avis favorable à l'impression :

Le livre des Proverbes de Salomon a toujours été regardé comme un des plus difficiles de la Sainte Écriture, tant à cause des expressions obscures et figurées qui en couvrent le sens, que parce qu'il est très mal aisé de trouver la liaison entre la plupart des sentences dont il est composé ${ }^{35}$.

20 L'hermétisme de cette poésie n'a rien d'accidentel; il ne s'explique pas non plus seulement par les mœurs et le goût des Hébreux : il est voulu par Dieu et obéit à des fins pédagogiques; le texte énigmatique convient en effet à notre état morbide, explique Sacy, et contribue à nous guérir de notre orgueil : «les hommes ont de la peine à souffrir cette obscurité [...] [Le Livre des Proverbes] ne nous instruit pas seulement comme des disciples, mais comme des malades ${ }^{36} »$. Le recours poétique aux " paraboles » fonctionne ainsi comme un chiffre destiné à crypter le message divin. La langue sacrée exige du chrétien qu'il s'abaisse devant les difficultés du texte, et qu'il tâche humblement de les éclaircir, avec l'aide de la grâce divine. De plus, comme les psaumes, les Proverbes se caractérisent par leur économie verbale; dix ans avant de convoquer l'exemple du Fiat Lux, comme ils le feront en 1682 dans la préface de la Genèse, les Messieurs considèrent déjà que la parole de Dieu dans les Proverbes se caractérise par une brevitas qui enclot de profonds mystères :

Il faut être Dieu pour parler si simplement, et en même temps si hautement et si divinement de toutes choses, et pour renfermer en si peu de mots une si grande étendue de sens, de mystères, et de vérités. ${ }^{37}$ 
comme les psaumes, les Proverbes sont aisés à apprendre, propres à imprégner la mémoire et, de là, à entrer dans l'âme; c'est pourquoi il faut en particulier les enseigner aux enfants, afin que « leur mémoire en étant remplie, ils [les proverbes] prissent en quelque sorte racine dès leurs premières années dans leur esprit et dans leur cœur ${ }^{38}$. » L'Ecclésiaste, également attribué à Salomon, présente aussi cette élévation sublime doublée d'obscurité : «Ce livre est en quelque chose plus spirituel que celui des Proverbes [...] Il y a divers endroits dans ce livre qui sont fort obscurs ${ }^{39} »$. Reste le livre de Job, dont la nature poétique est indubitable pour les éditeurs :

Il est bon aussi de remarquer en passant, que dans la langue originale toute cette conférence qu'eut Job avec ses amis n'est point en prose, mais en vers. Et l'on sait assez qu'il est naturel à la poésie d'user d'expressions vives et hyperboliques, dont le sens doit être entendu par rapport à la disposition de celui parle; et non à la force de ces mêmes expressions qui tiennent toujours quelque chose de l'hyperbole ${ }^{40}$.

Cantique aux Proverbes, nous voyons ici se dessiner une unité entre les livres attribués à Salomon : l'obscurité cultivée, l'usage de figures hardies, une densité de sens qui confine au sublime constituent comme les marques du style poétique prêté au roi d'Israël. Ces procédés, qui définissent la poésie biblique aux yeux des Solitaires, se retrouvent encore dans les cantiques qui émaillent les livres historiques, comme le Chant de l'Exilé déjà évoqué, bien identifié comme poème, et dont le fonctionnement repose ici explicitement sur la répétition :

Moïse chanta ce cantique avec les principaux des Israélites ; soit que l'ayant reçu de Moïse par écrit, ils le chantassent après lui verset à verset ; soit qu'il continuât lui seul tout le cantique, et que le peuple répétât toujours le premier verset. Car c'est ainsi qu'il y a de certaines paroles qui se répètent toujours dans le Cantique des Trois jeunes hommes, et dans quelques psaumes. Et l'Église en use de cette sorte en quelques-uns de ses offices, comme à la consécration d'un temple ou d'un autel ${ }^{41}$.

Le Cantique de Déborah de même ${ }^{42}$, également défini comme poème (Déborah «va chanter un Cantique à la gloire du Seigneur »), se caractérise par son "obscurité » particulièrement impénétrable: "Tous les interprètes conviennent qu'il n'y a guère de passage de l'Écriture plus obscur, et dont le sens soit plus indéterminé que celui-ci ». "Déborah était prophétesse et remplie du Saint-Esprit », "inspirée de Dieu », et son poème « renfermait une prophétie touchant Jésus-Christ, mais assez obscure, et qui ne pouvait s'entendre sans un grand éclaircissement $[\ldots]^{43}$. »

Nous disposons maintenant d'une vision plus précise de la conception de la poésie biblique telle que les Messieurs l'envisage. Moins sensibles à la prétendue simplicité de la Bible qu'à sa richesse, ils y discernent une dimension lyrique et musicale («chant, cantique ») ; ils sont particulièrement réceptifs à l'audace, jugée tout orientale, dans l'emploi des figures, au premier rang desquelles la métaphore ; mais à leurs yeux, la poésie des Hébreux se définit aussi par une propension à l'hermétisme, la facilité de mémorisation liée à l'emploi de répétitions, un style sentencieux, et cette concision qui inscrit dans le langage humain la toute-puissance du Verbe de Dieu. Aux yeux des préfaciers, la poésie biblique, émanation de l'Esprit, détient comme la grâce dont elle est issue le pouvoir de charmer et de dominer les âmes : les psaumes sont, aux dires des éditeurs, les «armes les plus puissantes» dont dispose l'Église ${ }^{44}$. La sincérité de la poésie de David et Salomon n'exclut pas, au contraire, un style recherché et volontiers obscur : ces opacités procèdent de la stratégie, tant pédagogique que gnoséologique, 
d'accommodation ou de condescendance de Dieu à la situation présente de l'homme pécheur.

Il est temps d'en venir à Pascal. Car cette définition particulière de la poésie biblique, telle que l'envisagent les éditeurs de la Bible de Port-Royal, éclaire la conception pascalienne des Écritures : le Livre saint, tel que l'apologiste le présente au libertin dans les liasses des Pensées consacrées aux preuves du christianisme, obéit en effet à ce régime " poétique » d'interprétation du Texte sacré.

La poésie biblique occupe une place essentielle chez Pascal : Philippe Sellier a montré que si celui-ci cite peu les sapientiaux et jamais le Cantique, il est en revanche nourri des psaumes ${ }^{45}$. Or, aux yeux des éditeurs de la Bible, les poèmes composés par David contiennent l'ensemble de la Révélation (ils forment une "théologie parfaite»), mais brouillée par un nuage de figures. De même, estime Pascal, la Bible mêle les ombres à la clarté : Dieu s'y trouve « caché en partie et découvert en partie »(S.690). Elle exprime tantôt clairement, tantôt obscurément les principaux événements liés à l'histoire du peuple hébreu (le plus souvent énigmatiques, ils sont parfois «représentés à avec beaucoup de clartét $\left.e^{46}\right)$.

Pascal trouve un modèle herméneutique d'approche du divin dans cette dualité de la poésie sainte, qui conjoint à la simplicité biblique (la « simplicité des Évangiles », S. 240) la confusion des ténèbres presque impénétrables («obscurités [...] bizarres », S. 251). C'est en effet pour adapter son Être à l'état d'aveuglement de l'homme pécheur, incapable de soutenir l'éclat de la Vérité, que Dieu a élaboré cette stratégie de communication du mystère, suggère Pascal (S. 182) : «l'œil de l'homme voyait alors [dans son état originel] la majesté de Dieu ». Pascal a d'abord poursuivi, avant de rayer ces mots: "mais il n'a pu supporter... ». L'éclat de la majestas est en effet devenu insoutenable après le péché. Désormais, «c'est une maladie naturelle à l'homme de croire qu'il possède la vérité directement", prévient Pascal au seuil de L'Esprit de géométrie, alors que cet accès immédiat au Vrai nous est fermé du fait de notre faiblesse. Dieu, toutefois, dans son infinie bonté, n'a pas voulu nous laisser dans ce complet abandon. Il a choisi pour se faire connaître de se "proportionner » à nos défaillances : «La seule religion chrétienne est proportionnée à tous », estime ainsi Pascal (S. 252), qui précise les modalités de cette condescendance divine :

S'il n'y avait point d'obscurité, l'homme ne sentirait pas sa corruption. S'il n'y avait point de lumière, l'homme n'espérerait point de remède. Ainsi il est non seulement juste, mais utile pour nous, que Dieu soit caché en partie. (S. 690)

La théologie du "Deus absconditus» (S. 275) correspond ainsi à une économie de la Révélation qui repose sur l'accommodation de Dieu à la misère des hommes. Dieu, qui tout à la fois se donne à voir et se dissimule partiellement, condescend par-là, sur le modèle d'une Incarnation conçue comme kénose, à abaisser sa dignité jusqu'à employer des figures inadéquates pour se faire entendre des hommes; il accepte, pour notre profit, de tempérer l'enargeia de la Révélation sous le voile de figures dont la fonction est épistémologique ${ }^{47}$. Celles-ci ont permis de «rendre le Messie connaissable aux bons ", et de leur faire découvrir cette "intelligence des biens promis », qui « dépend du cœur » (S. 287). Cette "obscurité » est donc bénéfique aux élus, mais elle sert également à la damnation des réprouvés dont l'âme de pierre ne franchit pas les voiles qui préservent la dignité divine ${ }^{48}$.

En tant qu'art du détour et voie indirecte menant au Vrai, la poésie occupe une place essentielle dans cette stratégie de communication du divin: le clair-obscur qui la 
caractérise constitue, plus que toute autre forme de parole, un mode d'accès à l'Être qui ne se laisse pas enfermer dans l'étroit corset de la logique et du discours rationnel. Si Pascal, et tout Port-Royal, considère le psautier comme un livre exceptionnel ${ }^{49}$, c'est parce que, du fait de sa nature poétique, il mobilise plus que toute autre partie de la Bible la vaste panoplie des procédés figuratifs susceptibles d'évoquer dans le trouble diaphane de son " genre nouveau d'écrire ${ }^{50}$ » cet incompréhensible divin qui « ne laisse pas d'être ». L'importance gnoséologique de la poésie est attestée en particulier par le fragment 303, où Pascal formalise le lien entre figures poétiques et connaissance; l'accommodation passe par le biais d'un usage poétique de la langue :

Quand la parole de Dieu, qui est véritable, est fausse littéralement, elle est vraie spirituellement. Sede a dextris meis : cela est faux littéralement, donc cela est vrai spirituellement.

En ces expressions il est parlé de Dieu à la manière des hommes. Et cela ne signifie autre chose sinon que l'intention que les hommes ont en faisant asseoir à leur droite, Dieu l'aura aussi. C'est donc une marque de l'intention de Dieu, non de sa manière de l'exécuter.

Ainsi quand il dit: Dieu a reçu l'odeur de vos parfums et vous donnera en récompense une terre grasse, c'est-à-dire la même intention qu'aurait un homme qui, agréant vos parfums, vous donnerait en récompense une terre grasse. Dieu aura la même intention pour vous parce que vous avez eu pour lu[i la] même intention qu'un homme a pour celui à qui il donne des parfums.

Ainsi iratus est, Dieu jaloux, etc. Car les choses de Dieu étant inexprimables, elles ne peuvent être dites autrement et l'Église aujourd'hui en use encore, quia confortavit seras, etc. (S. 303)

La colère de Dieu, qui apparait à plusieurs reprises dans la Bible ${ }^{51}$, est un lieu topique de l'exégèse, et l'occasion de mises au point patristiques essentielles sur la notion de condescendance. Les Pères s'accordaient en effet à considérer que la colère ne saurait constituer l'attribut d'un Dieu dont l'essence est au contraire l'impassibilité : « Dieu est bon, impartial et immuable. [...] Dieu ne se réjouit ni ne se met en colère, car se réjouir et être offensé sont des passions ", précise ainsi saint Antoine le Grand ${ }^{52}$; Jean Cassien, de même, considère que «lorsque nous lisons les mots de "colère de Dieu", nous devons nous garder de les interpréter comme s'il s'agissait d'une passion humaine ${ }^{53} »$. En réalité, commente encore Cyrille d'Alexandrie, « l'Essence divine n'est sujette à aucune de ces passions [courroux et colère], mais ressent une espèce d'indignation qui n'est connue que de Dieu seul et qui est proprement inexprimable $e^{54} »$. Pascal justifie de même par la condescendance les métaphores bibliques: «En ces expressions il est parlé de Dieu à la manière des hommes ${ }^{55}$ »; l'auteur des Pensées paraphrase ici saint Paul («je vous parle humainement à cause de la faiblesse de votre chair »)56, mais se souvient aussi de la question 52 de saint Augustin : «Les divines Écritures pour nous élever du sens terrestre et humain jusqu'au divin et céleste, ont condescendu à ce langage qui est d'usage familier même entre les gens les plus incultes ${ }^{57}$ ».

On ne saurait se dispenser d'user des métaphores car l'Essence ineffable de Dieu s'élève au-delà de tout discours rationnel. La métaphore, dont on a vu qu'elle était constitutive de la poétique des psaumes en particulier, est ainsi un pis-aller certes, mais nécessaire pour évoquer "l'inexprimable " majesté de Dieu. Des images familières et triviales, comme celles sur lesquelles Pascal appuie sa démonstration («asseyez-vous à ma droite ", Ps 109, 1), sont meilleures que celles qui tenteraient maladroitement de cerner la nature divine : la "colère de Dieu » est si évidemment inadéquate qu'elle révèle d'emblée son insuffisance essentielle, sauf à donner dans un naïf anthropomorphisme, ou plutôt une anthropopathie ${ }^{58}$. Les métaphores psalmiques ou les images poétiques, 
comme celle tirée de la Genèse et mentionnée dans le fragment 303, permettent, avec toutes les limites qui sont les leurs, de suggérer la réalité de l'Être indicible : on ne peut la dire "autrement ", et surtout pas en usant de ces inutiles "mots d'enflure ", ni de ces expressions faussement « hautes, élevées, sublimes » que réprouve Pascal à la fin de L'esprit géométrique et de l'art de persuader ${ }^{59}$. De même, dans les Pensées, il déclare son mépris pour la mauvaise poésie qui dit "de petites choses avec de grands mots ${ }^{60}$ ", au rebours de la parole divine. Les métaphores « basses communes, familières », telles que celles choisies par David, "conviennent mieux " à la vérité, et manifestent mieux l'abaissement d'un Dieu qui s'humilie jusqu'à accepter les imperfections de la langue humaine; ces tropes apparaissent ainsi au cœur de l'herméneutique figurative que propose Pascal dans les Pensées. Le paradigme poétique propose en effet une appréhension non discursive des réalités divines, qui remédie à la disqualification de la raison consécutive au péché : les « énigmes » de Job ou des Proverbes sont propres à la fois à éprouver l'humilité du libertin et à le « réveiller » de sa torpeur ${ }^{61}$, mais surtout à lui faire découvrir des vérités essentielles qui échappent à cette raison dont il fut naguère idolâtre. Pascal généralise ainsi un rapport aux textes bibliques qui convient en fait essentiellement aux textes poétiques : la figure, dans son ambiguité, portant absence et présence, plaisir et déplaisir, relève d'un fonctionnement métaphorique de la langue.

Mais connaître la Vérité ne suffit pas : «qu'il y a loin de la connaissance de Dieu à l'aimer " (S. 409). La poésie, si elle participe comme on l'a vu de l'intelligence de la Révélation, se révèle aussi utile pour attirer les âmes à la Charité. En effet, d'après Pascal, la poésie se définit par son objet, qui est «l'agrément » (S. 486). Or, les âmes sont ici-bas si corrompues qu'il n'est plus pour elle de chemin vers l'Amour qui ne passe par le plaisir :

Et c'est pour punir ce désordre par un ordre qui lui est conforme, que Dieu ne verse ses lumières dans les esprits qu'après avoir dompté la rébellion de la volonté par une douceur toute céleste qui le charme et qui l'entraîne ${ }^{62}$.

La poésie, en tant que par essence elle confère un plaisir, apparait donc comme une voie offerte à la créature pour s'élever jusqu'à l'Amour de Dieu.

Les psaumes de David, une nouvelle fois, constituent le modèle de cette bonne poésie : aux yeux de Port-Royal, ils sont en mesure de vaincre les résistances de l'âme, et de la porter à cette sainte délectation qui seule sera capable d'émouvoir notre volonté; la musique des psaumes envoûte l'esprit, et attire le pécheur comme malgré lui vers le Ciel : la poésie "charme les oreilles", par "une certaine douceur qu'elle inspire à l'âme ${ }^{63}$ ", et entraîne par là les hommes sur le chemin de la Charité. David en effet " ne tend pas, par les sons sacrés qu'il forme, à plaire seulement à nos oreilles; mais à procurer un plaisir et un bien solide à nos âmes ${ }^{64} »$; pour se mettre à la mesure de notre péché, Dieu « qui seul peut mettre [ses vérités] dans l'âme, et par la manière qu'il lui plaît [...] a voulu qu'elles entrent du cœur dans l'esprit ", c'est-à-dire qu'elles passent par la volonté avant de toucher la raison. Ce mode de transmission de la vérité est précisément celui qu'emploie David : les psaumes font « entrer par l'oreille dans le cœur la connaissance de la vérité qui doit affermir notre salut ${ }^{65} »$.

La volupté sainte que confère la poésie des psaumes, et dans laquelle il faut voir un itinéraire vers l'Amour, contribue ainsi à expliquer le souvenir omniprésent des versets de David dans l'écriture pascalienne. 
Certes, l'accommodation, entendue dans ce sens épistémologique, convient à Dieu seul, et on conçoit qu'il ne soit point facile de la réduire en techniques susceptibles d'être facilement reproduites. Mais c'est précisément la souplesse et la malléabilité de la poésie hébraïque, dans laquelle on ne reconnaît pas de forme fixe, de contraintes prosodiques ou de règles de métrique, qui va faciliter son appropriation au sein d'une prose qu'habite la quête de la grâce. Je ne m'étendrai pas longuement ici : plusieurs lecteurs éminents de Pascal ont montré avec succès comment celui-ci avait pressenti certaines mécaniques propres à la rhétorique sémitique, et qui ne seraient formalisées que bien plus tard, voire tout récemment. Ainsi, Philippe Sellier n'évoque jamais le fragment 339 que comme un "poème ${ }^{67}$ ». Olivier Jouslin a montré qu'on pouvait regarder Pascal comme un poète en prose préfigurant une écriture du verset qui n'émergera qu'au $\mathrm{xx}^{\mathrm{e}}$ siècle ${ }^{68}$. Les travaux tout récents de Laurent Susini ${ }^{69}$, qui s'appuient efficacement sur l'ambitieux appareil théorique élaboré par Roland Meynet ${ }^{70}$, ouvrent une nouvelle voie prometteuse d'exploration, que confirme encore la présente journée d'étude. Par imprégnation, Pascal pouvait avoir été bien plus lucide qu'on ne le dit sur l'importance matricielle du parallélisme ou de la synonymie ${ }^{71}$. Philippe Sellier a montré que l'apologiste affectionne la construction parallèle à deux ou trois membres : «Le procédé que les exégètes de la Bible appellent "parallélismes hébraïques" est omniprésent dans l'Apologie ${ }^{72} »$. Et le critique de s'appuyer sur plusieurs fragments bâtis selon ce principe, comme cet extrait du fragment S. 78 : «Rien ne lui montre la vérité. Tout l'abuse »; ou encore le début du fragment S. 221 :

Nous ne connaissons Dieu que par Jésus-Christ.

Sans ce Médiateur est ôtée toute communication avec Dieu.

Par Jésus-Christ nous connaissons Dieu.

Pascal emprunte également beaucoup aux figures capables d'émouvoir et "remuer " son lecteur. Le fait est connu, et n'exige pas de nouvelle démonstration, sauf à rappeler que ces procédés caractérisent tout particulièrement aux yeux de Port-Royal l'écriture des psaumes et des cantiques : ainsi on trouve chez l'apologiste bien des exemples de l'hyperbole, qui ressortit au movere dans la rhétorique classique, mais qui constitue aussi d'après les Solitaires l'un des caractères de la poésie hébraïque. L'énigme, les sentences, ou le souci de se faire citer et mémoriser, dont l'importance chez Pascal a déjà été bien repérée, correspondent en fait à des traits qui renvoient aux psaumes : la sentence proverbiale en effet, aux dires de Sacy, convient à l'état de malade des libertins, parce qu'elle parle selon le besoin et non selon le désiri3. Pascal goûte enfin, comme David et Salomon, le style familier, et les sujets pris aux « entretiens ordinaires de la vie » (S 618). L'apologiste allègue ici Montaigne et Épictète, mais pour évoquer aussitôt après la figure de Salomon. 


\section{Conclusion}

Pascal se défie de la mauvaise poésie. Le fragment "Beauté poétique " dénonce l'affectation de vers précieux ou maniéristes, au mauvais sens qu'on donne volontiers à ces termes, c'est-à-dire tout d'enjolivements fades et mièvres. De cette diatribe contre les «reines de village » formulée dans le fragment 486, il ne faudrait pas conclure toutefois à une condamnation de toute espèce de poésie : aux jeux de langage vains et inutiles de versificateurs virtuoses, Pascal oppose l'authenticité d'une Parole divine qui, structurellement, ne peut s'exprimer qu'à travers un usage poétique de la langue, et singulièrement des métaphores. Les obscurités et les énigmes, les familiarités, les tropes, et l'agrément invincible d'une musique céleste capable comme la grâce de charmer les cœurs: tels sont les truchements par lesquels Dieu s'accommode à la misère des hommes, à la fois pour les attirer à lui et pour se faire connaître à eux. Mais Pascal ne cantonne pas cette écriture diaphane aux seuls livres poétiques de la Bible: c'est tout le Texte sacré qui obéit à ce régime d'écriture en clair-obscur et devient ainsi un vaste code énigmatique dont Jésus-Christ fournit la clef. La trajectoire que propose Pascal au libertin dans la seconde partie de l'Apologie passe ainsi nécessairement par une lecture poétique des Écritures : c'est en délaissant le discours rationnel au profit d'une langue assombrie de figures que le lecteur apprend à connaître et goûter la resplendissante lumière d'une Vérité dont le propre est d'embrasser les " contrariétés » - une vérité essentiellement poétique, et à ce titre, à la fois "vénérable » et " aimable » (S. 46).

\section{NOTES}

1. Nous adoptons le classement de Philippe Sellier (Paris, Bordas, 1991), noté S. suivi du numéro du fragment.

2. Olivier Jouslin, "Pascal poète en prose ", Dix-septième siècle, vol. 221, $\mathrm{n}^{\circ} 4,2003$, p. 715-747.

3. Philippe Sellier, «Rhétorique et apologie : Dieu parle bien de Dieu », dans Port-Royal et la littérature. Pascal [1999], Paris, Éditions Honoré Champion, « Champions classiques Essais », 2010, p. 239-250 ; Laurent Susini, « La "vraie éloquence" en question dans les Pensées de Pascal », Revue d'histoire littéraire de la France, vol. 103, n 1 1, 2003, p. 17-29; voir aussi, du même auteur : L'Écriture de Pascal. La lumière et le feu : la "vraie éloquence » à l'œuvre dans les Pensées, Paris, Honoré Champion, «Lumière classique », 2008.

4. La Rhétorique ou l'art de parler [1675], éd. Christine Noille-Clauzade, Paris, Champion, "Sources classiques », 1998, chapitre XXI, p. 313. L'opinion, envisagée par Lamy, selon laquelle il n'y aurait pas de spécificité des livres prétendus bibliques trouve encore aujourd'hui des partisans; ainsi Douglas K. Stuart estime ainsi dans Studies in early Hebrew Meter (Harvard, Scholar Press, 1976) que la prétendue "poésie » biblique ne serait qu'une prose particulièrement ornée et figurée. 
5. Robert Alter, L'art de la poésie biblique [1985], traduit de l'anglais par Christine Leroy et Jean-Pierre Sonnet, Bruxelles, Lessius, coll. Le livre et le rouleau, 11, 2003.

6. Lancelot est l'auteur de Quatre traités de poésies latine, française, italienne et espagnole, Paris, Pierre Le Petit, 1663. Pierre Nicole a écrit une Dissertatio de vera pulchritudine et adumbrata, placée en tête d'un Epigrammatum Delectus paru en 1659; on lui attribue aussi la préface du Recueil de poésies chrétiennes et diverses (Paris, Pierre Le Petit, 1671), anthologie composée par des proches de Port-Royal et signée par Jean de La Fontaine.

7. L'Exode et le Lévitique traduits en français [1682], Paris, Guillaume Desprez et JeanBaptiste Desessars, 1701, chapitre X, « sens littéral », p. 214.

8. Ibid.

9. Le Cantique des cantiques traduit en français [1693], Paris, Guillaume Desprez, 1694, «Préface », non paginée.

10. Ibid.

11. Ibid.

12. Selon eux, même le livre de Job ne doit pas être vu comme une " fiction poétique ", au sens de fiction vraisemblable (Le Livre de Job traduit en français [1688], Paris, Guillaume Desprez, 1695, "Préface»). "Cette histoire ne peut être, comme certains l'ont cru, une simple parabole ", précise le préfacier.

13. Le Cantique des cantiques traduit en français, op. cit., « Préface » non paginée.

14. Cf. 1 Co. $13,12$.

15. Le Cantique des cantiques traduit en français, op. cit., « Préface » non paginée.

16. Quintilien, Institution oratoire, livre II, chap. 8.

17. Le Livres des psaumes traduit en français [1689], Bruxelles, Henry Fricx, 1699, t. I, «Préface », p. XXXII.

18. Ibid., «Préface », p. XXX.

19. Ibid., «Préface », p. XXXII.

20. Ibid., « Préface », p. XXVI.

21. Ibid., « Préface », p. XXX.

22. C'est le sens que Furetière donne à "charme", quand ce terme ne signifie pas enchantement.

23. Le Livres des psaumes traduit en français, op. cit., "Préface », p. XX.

24. Sur l'histoire de la publication et plus généralement la place des psaumes à PortRoyal, voir en particulier Bernard Chédozeau, Port-Royal et la Bible. Un siècle d'or de la Bible en France (1650-1708), Nolin, « Univers Port-Royal », p. 77-169.

25. Le Livre des psaumes, op. cit.., "Préface », p. XXIX.

26. Ibid., «Préface », p. XIX.

27. Ibid., « Préface », p. XXXIV.

28. Ibid., « Préface», p. XXXV.

29. Ibid., " Préface », p. XX.

30. Ibid., « Préface », p. XXXII.

31. Ibid., «Préface », p. XIX.

32. Ibid., « Préface », p. XXXIII. 
33. Ibid.

34. Les Proverbes de Salomon traduits en français [1672], Paris, Guillaume Desprez et JeanBaptiste Desessars, 1711, « Préface » non paginée.

35. Ibid., « Approbation de Monseigneur l'évêque d'Alet ».

36. Ibid., « Préface ».

37. Ibid.

38. Ibid., « Préface ».

39. L'Ecclésiaste de Salomon traduit en français [1673], Paris, Guillaume Desprez et JeanBaptiste Desessars, 1711, « Préface ».

40. Le Livre de Job traduit en français, op. cit., « Préface ».

41. L'Exode et le Lévitique traduits en français, op. cit., p. 213-214.

42. Le Livre des Juges traduits en français [1687], Bruxelles, E. H. Fricx, 1713, p. 73, «Sens littéral et sens spirituel $»$.

43. Ibid.

44. Les Psaumes de David traduits en français, op. cit., " Préface », p. XXVI.

45. Philippe Sellier, «La Bible de Pascal », in Port-Royal et la littérature [1999], Paris, Librairie Honoré Champion, Champions Classiques, Essais, 2010, p. 203.

46. Les Psaumes traduits en français, op. cit., p. XXXI.

47. De Doctrina Christiana, I, 13. Voir sur ce sujet Arnold Huigen, Divine Accommodation in John Calvin's Theology: Analysis and Assessment, p. 88. Voir aussi De Vera Religione, 17, 34.

48. S. 271.

49. « Au lieu que les livres des prophètes instruisent d'une manière, et les livres historiques d'une autre manière ; que la Loi a ses instructions particulières, et que les Proverbes enseignent aussi d'une autre façon; le livre des psaumes renferme lui seul tout ce qu'il y a d'utile dans tous les autres » (Les Psaumes de David traduits en français, op. cit., « Préface », p. XXXI).

50. Les Psaumes de David traduits en français, op. cit., "Préface », p. XXX.

51. Par exemple Ps. 7, 12.

52. Philocalie, De la Conduite vertueuse, 150.

53. Saint Jean Cassien, Institutes, livre VIII, IV.

54. Saint Cyrille d'Alexandrie, Commentaire sur saint Jean, livre IX, XIII.

55. Pensées, fr. S. 303.

56. « Humanum dico, propter infirmitatem carnis vestrae », Rom. 6, 19.

57. "Divinae Scripturae a terreno et humano sensu ad divinum et coelestem nos erigentes, usque ad ea verba descenderunt, quibus inter se stultissimorum etiam utitur consuetudo", Quatre-vingt-trois questions diverses, question 52.

58. On désigne sous le nom d'anthropopathie la figure par laquelle on attribue à Dieu des passions ou des sensations qui n'appartiennent qu'aux hommes.

59. L'Esprit géométrique, II, De l'art de persuader, § 30, in Blaise Pascal, Euvres complètes, Paris, Desclée de Brouwer, 1992, t. III, p. 428.

60. Pensées, fr. S. 486. 
61. "Primo ipsa invectio, quasi sopitis sensibus ut evigilarent, que fremiu illisa est?" (Augustin, De Doctrina christiana, IV, VII, 17).

62. De l'Art de persuader, $\S 4$, p. 414.

63. Les Psaumes de David traduits en français, op. cit., " Préface », p. XXXII.

64. Ibid., p. XXX.

65. Ibid., p. XXX.

66. B. Pascal, « De l'Esprit géométrique », II, De l'art de persuader, § 10, p. 416.

67. Philippe Sellier, « Rhétorique et apologie : 'Dieu parle bien de Dieu' », in Port-Royal et la littérature, op. cit., p. 124.

68. Art. cit.

69. Laurent Susini, « Pour un Pascal juif. Ordre du cœur et rhétorique sémitique dans l'œuvre pascalienne ", in Retorica biblica e semitica 3. Atti del terzo convegno RBS, sous la direction de R. Meynet et J. Oniszczuk, Bologne, EDB, 2013, p. 317-345.

70. Voir en particulier son Traité de rhétorique biblique, Paris, Lethielleux, « Rhétorique sémitique ", 2007.

71. Ces tournures étaient trop habituelles dans la Bible pour n'avoir jamais été pressenties : "Les antithèses ont plus de poids en hébreu qu'en grec ou en latin. Voici qui serait froid en grec ou en latin : 'cet homme est stupide, et sans intelligence' [Jer. 4, 22]. Le second membre semble en effet enlever quelque chose. Mais l'hébreu procède autrement ", écrit ainsi Calvin. Texte cité par Olivier Millet, Calvin et la dynamique de la parole. Essai de rhétorique réformée, Paris, Librairie Honoré Champion, 1992, p. 385. Quelques décennies après Pascal, Le Sesne d'Etemare bâtira ses Gémissements sur semblables procédés de parallélismes calqués sur ceux de la Bible (voir " Les Gémissements de l'abbé d'Étemare, ou la poésie des ruines ", in Chroniques de Port-Royal, $\mathrm{n}^{\circ}$ 55, 2005, p. 143-168).

72. Philippe Sellier, « Rhétorique et apologie », art. cit., p. 124.

73. Livre des Proverbes traduit en français, préface, p. X.

INDEX

Mots-clés : poésie, Bible, Pensées

Keywords : poetry, Bible, Pensées

\section{AUTEUR}

\section{TONY GHEERAERT}

Professeur de littérature française du xviie siècle, CÉRÉdI, Université de Rouen 